\title{
Analysis of changes in the expression of Notch1 and HES1 and the prognosis of osteosarcoma patients following surgery
}

\author{
LONG FANG $^{1}$, BEI LI ${ }^{1,2}$, DAPENG YU ${ }^{3}$, BAOLONG WANG ${ }^{1}$ and TINGBAO ZHAO ${ }^{1}$ \\ ${ }^{1}$ Department of Orthopaedics, Shangdong Provincial Third Hospital, Cheeloo College of Medicine, Shangdong University; \\ ${ }^{2}$ Department of Orthopaedics, Shandong Cancer Hospital and Institute, Shandong First Medical University and \\ Shandong Academy of Medical Sciences; ${ }^{3}$ Department of Orthopaedics, Shangdong \\ Provincial Western Hospital, Jinan, Shandong 250000, P.R. China
}

Received September 20, 2019; Accepted June 4, 2020

DOI: $10.3892 / 01.2020 .11890$

\begin{abstract}
The present study aimed to analyze the changes in the expression of Notch1 and hairy and enhancer of split-1 (HES1) and the prognosis of patients with osteosarcoma following surgery. Samples from 62 patients with osteosarcoma treated at Shandong Cancer hospital from April, 2011 to June, 2013 were collected as the research group, and those from 52 healthy individuals undergoing physical examination were collected as the control group. The expression levels of Notch1 and HES1 in the serum of patients with osteosarcoma were measured by ELISA before and after surgery. Pearson's correlation analysis was used to analyze the correlation between Notch1 expression and HES1 expression in the osteosarcoma patients. According to the expression levels of Notch1 and HES1, the patients were divided into the high expression group and the low expression group, and the 5-year survival rate of the patients was observed. The expression levels of Notch1 and HES1 in the osteosarcoma patients before surgery were higher than those after surgery $(\mathrm{P}<0.05)$. The sensitivity, specificity and AUC of Notch1 for osteosarcoma were 93.55\%, 58.06\% and 0.732 respectively, and those of HES1 were $82.26 \%, 61.29 \%$ and 0.766 , respectively. The expression level of Notch1 positively correlated with the expression level of HES1 in the osteosarcoma patients $(\mathrm{r}=0.795, \mathrm{P}<0.001)$. According to the expression levels of Notch1 and HES1, the patients were divided into the high and low expression groups. The survival rate of the low expression group was significantly higher than that of the high expression groups $(\mathrm{P}=0.045)$. Finally, multiple factors were analyzed by logistic regression, and it was found that tumor location, chemotherapy response, tumor size, Notch1 and HES1 were independent risk factors
\end{abstract}

Correspondence to: Dr Tingbao Zhao, Department of Orthopaedics, Shangdong Provincial Third Hospital, Cheeloo College of Medicine, Shangdong University, 11 Wuyingshan Road, Jinan, Shandong 250000, P.R. China

E-mail: daos68@163.com; doctorzhaotingbao@163.com

Key words: osteosarcoma, Notch1, HES1, prognosis analysis for prognosis. Notch1 and HES1 exhibited a low expression in patients following surgery. ROC curve analysis revealed that the two indicators had good diagnostic efficacy and were expected to become markers for diagnosis and prognosis of osteosarcoma.

\section{Introduction}

Osteosarcoma is the most common malignant tumor among children, adolescents and young adults (1). Osteosarcoma originates from primary bone-forming mesenchymal cells, accounting for $20 \%$ of all primary osteosarcoma, and is the most common primary bone malignant tumor (2). Osteosarcoma usually occurs in the long bone of limbs near the metaphyseal plate. The most common sites are the femur, tibia and humerus (3). Before 1970, the treatment for osteosarcoma was mainly surgical resection. With the application of multi-drug regimens, chemotherapy has markedly improved the 5-year survival rate of patients with localized osteosarcoma from $<20$ to $65 \%$; however, its prognosis is still very poor (4). Moreover, the mortality rate of patients with recurrent and metastatic osteosarcoma is still very high. Therefore, it of utmost importance to explore novel prognostic factors for osteosarcoma patients, particularly those diagnosed with metastatic disease.

Notch1 is a type 1 transmembrane receptor protein, which is important for cell fate regulation, the differentiation of various systems and neuronal development, such as neurogenesis and the maintenance of neural stem cells (5). The increased expression of Notch1 is related to the low survival rate of patients with various types of cancer (6-8). The proliferation of cells from these types of cancer can be inhibited by the pharmacological inhibition of Notch1. Therefore, preventing the occurrence of Notch1 is a potential strategy for the treatment of various types of cancers (9). The transcription factor hairy and enhancer of split-1 (HES1) is a member of the basic helix-loop-helix (BHLH) of transcription inhibitor family, and is the downstream target of Notch signal pathway (10). HES1 is overexpressed in a number of tumor types, including colon cancer (11), breast cancer (12), non-small cell lung cancer (13), etc., suggesting that HES1 has carcinogenic activity and is closely associated with cancer. 
Therefore, the present study examined the changes in the expression of Notch1 and HES1 in osteosarcoma patients following surgery. The correlation between Notch1 expression and HES1 expression, and its association with prognosis were also investigated, so as to identify novel potential diagnostic and treatment targets that may be used clinical practice.

\section{Patients and methods}

General patient information. In the present study, samples from 62 patients with osteosarcoma treated at Shandong Cancer Hospital from April, 2011 to June, 2013 were collected as the research group, and those from 52 healthy individuals undergoing a physical examination were collected as the control group. There were 33 males and 29 females in the research group, with an average age of $18.6 \pm 10.1$ years, while the control group consisted of 28 males and 24 females with an average age of $19.1 \pm 10.3$ years. The present study was approved by the Ethics Committee of Shandong Cancer Hospital. Signed written informed consents were obtained from the patients and/or parents or guardians.

Inclusion and exclusion criteria. The inclusion criteria were as follows: Patients who met the ESMO diagnostic criteria (14), or received treatment at Shandong Cancer Hospital after diagnosis; patients who did not receive radiotherapy or chemotherapy prior to surgery; patients who did not receive any treatment within 30 days after surgery; patients aged between 10 to 40 years; patients with complete case data; patients who agreed to cooperate with the work arrangement of the medical staff at Shandong Cancer Hospital; patients or their immediate family members signed informed consents.

The exclusion criteria were as follows: Patients who died during the course of treatment; patients with injury to important organs; patients suffering from other cardiovascular and cerebrovascular diseases, as well as any physical disability; pregnant mothers; patients suffering from other autoimmune diseases and chronic diseases; patients transferred to Shandong Cancer Hospital; patients with contraindications to surgery, mental diseases and language dysfunction.

Surgical treatment plan. The patients were subjected to limb preservation surgery according to the strategies outlined in the study by Ando et al (15) and references listed in that study.

Blood sample processing. Before surgery and at 30 days after surgery, early in the morning on an empty stomach, venous blood was drawn and stored at $4^{\circ} \mathrm{C}$ for $30 \mathrm{~min}$, and the serum samples were then centrifuged for $10 \mathrm{~min}$ at $4^{\circ} \mathrm{C}(1,500 \mathrm{x} \mathrm{g})$. The supernatant was then extracted and stored in a refrigerator at $-80^{\circ} \mathrm{C}$.

Main reagents. Notch1 and HES1 kits were purchased from Wuhan Feien Biotechnology Co., Ltd. (cat. nos. EH0926 and EH3223), and were used strictly in accordance with the operating instructions provided with the kits. The Eppendorf CryoCube F740hi ultra-low temperature refrigerator was purchased from Eppendorf Co., Ltd. (cat. no. ep000000).
Follow-up of patients. The patients were followed-up for 5 years, and their survival rates were recorded via telephone communications and outpatient medical records. The follow-up time points were the $3 \mathrm{rd}, 6,9$ and 12 th month of each year.

Observation indicators. The main observation indicators were as follows: The expression levels of Notch1 and HES1 in osteosarcoma patients before and after surgery were observed, and the diagnostic value of Notch1 and HES1 in osteosarcoma was determined.

The secondary observation indicators were the following: Pearson's correlation analysis was used to analyze the correlation between Notch1 expression and HES1 expression in osteosarcoma patients. According to the expression levels of Notch1 and HES1 (obtained by ELISA), the patients were divided into the high and low expression groups, and the 5-year survival rate of the patients was observed.

Statistical analysis. In the present study, the SPSS20.0 software package was used to perform the statistical analysis on the collected data. The GraphPad 7 software package was used to obtain the required graphs, and the Kolmogorov-Smirnov test was used to analyze the distribution of these data, in which normally distribution data were expressed as the mean \pm standard deviation (means \pm SD). Inter-group comparisons were conducted using an independent-samples t-test, and intra-group comparisons were conducted using a paired t-test. Count data are expressed as a percentage $(\%)$ and analyzed using the Chi-squared $\left(\chi^{2}\right)$ test. A receiver operating characteristic (ROC) curve was created to plot the diagnostic value of Notch1 and HES1 in osteosarcoma, which was represented by the $\chi^{2}$ value. Cut-off values were calculated using Youden's index (YI) calculation formula as follows: $\mathrm{YI}=[\mathrm{a} /(\mathrm{a}+\mathrm{c})+\mathrm{d} /(\mathrm{b}+\mathrm{d})]-1$. Pearson's correlation analysis was used to analyze the correlation between Notch1 expression and HES1 expression in the osteosarcoma patients. The 5-year survival of the patients was plotted by the Kaplan-Meier survival curve and data were analyzed using the log-rank test. In addition, univariate and multivariate logistic regression were performed to analyze the independent risk factors affecting the prognosis of the patients. $\mathrm{P}<0.05$ was considered to indicate a statistically significant difference.

\section{Results}

Clinical data. No significant differences were observed in the clinical data of the research group and the control group, including age, sex, body mass index (BMI), marital status, nationality, place of residence, smoking, alcohol consumption and exercise, which proved comparability $(\mathrm{P}>0.05)$, as shown in Table I.

Expression levels of Notchl and HES1 in osteosarcoma patients before and after surgery. The expression levels of Notch1 and HES1 in the osteosarcoma patients before surgery were $15.03 \pm 1.35$ and $13.86 \pm 1.53$, while the expression levels of Notch1 and HES1 in the osteosarcoma patients after surgery were $4.12 \pm 1.01$ and 5.02 \pm 0.99 , respectively. Significant differences were observed in the comparisons of Notch1 and HES1 
Table I. Clinical basic data of the patients.

\begin{tabular}{|c|c|c|c|c|}
\hline Characteristic & Research group $(\mathrm{n}=62)$ & Control group $(\mathrm{n}=52)$ & $\chi^{2}$ or t-test value & P-value \\
\hline Age (years) & $18.6 \pm 10.1$ & $19.1 \pm 10.3$ & 0.261 & 0.795 \\
\hline Sex, no. (\%) & & & 0.004 & 0.947 \\
\hline Male & $33(53.23)$ & $28(53.85)$ & & \\
\hline Female & $29(46.77)$ & $24(46.15)$ & & \\
\hline $\operatorname{BMI}\left(\mathrm{kg} / \mathrm{m}^{2}\right)$ & $22.26 \pm 0.37$ & $22.21 \pm 0.25$ & 0.828 & 0.409 \\
\hline Marital status, no. (\%) & & & 0.001 & 0.981 \\
\hline Married & $13(20.97)$ & $11(21.15)$ & & \\
\hline Unmarried & $49(79.03)$ & $41(78.85)$ & & \\
\hline Nationality, no. (\%) & & & 1.077 & 0.299 \\
\hline Han & $55(88.71)$ & $49(94.23)$ & & \\
\hline Minority & $7(11.29)$ & $3(5.77)$ & & \\
\hline Place of residence, no. (\%) & & & 0.129 & 0.719 \\
\hline Cities and towns & $32(51.61)$ & $30(48.39)$ & & \\
\hline Countryside & $30(48.39)$ & $32(51.56)$ & & \\
\hline Smoking history, no. (\%) & & & 0.500 & 0.480 \\
\hline Yes & $11(17.74)$ & $12(23.08)$ & & \\
\hline No & $51(82.26)$ & $40(76.92)$ & & \\
\hline Alcohol consumption history, no. (\%) & & & 1.273 & 0.259 \\
\hline Yes & $28(45.16)$ & $29(55.77)$ & & \\
\hline No & $34(54.84)$ & $23(44.23)$ & & \\
\hline Exercise habits, no. (\%) & & & 1.433 & 0.231 \\
\hline Yes & $30(48.39)$ & $31(59.62)$ & & \\
\hline No & $32(51.61)$ & $21(40.38)$ & & \\
\hline
\end{tabular}

expression levels before and after surgery $(\mathrm{P}<0.05)$, as shown in Fig. 1.

Diagnostic value of Notchl and HES1 expression in osteosarcoma. ROC curve analysis demonstrated that when the cut-off value was 13.230, the sensitivity, specificity and AUC of Notch1 in the diagnosis of osteosarcoma were $93.55 \%$, $58.06 \%$ and 0.732 , respectively $(\mathrm{P}<0.001)$; when the cut-off value was 12.810 , the sensitivity, specificity and AUC of HES1 in the diagnosis of osteosarcoma were $82.26 \%, 61.29 \%$ and 0.766, respectively $(\mathrm{P}<0.001)$, as shown in Table II and Fig. 2.

Correlation between Notchl and HES1 expression in patients with osteosarcoma. Pearson's correlation analysis identified that the expression level of Notch1 positively correlated with that of HES1 in the osteosarcoma patients $(\mathrm{r}=0.795, \mathrm{P}<0.001)$, 95\% CI: 0.681-0.872, as shown in Fig. 3.

High and low expression levels of Notchl and HES1, and the 5-year survival rate of patients with osteosarcoma. The patients in the present study were then divided into the Notch1 high expression group ( $\geq 16.57)$, the HES1 high expression group ( $\geq 15.18$ ) (31 cases), the Notch1 low expression group $(<16.57)$ and the HES1 low expression group $(<15.18)$ (31 cases) according to the median value of the expression levels of Notch1 and HES1. All the patients were interviewed at follow-up. In the Notch1 and HES1 low expression groups, 10 patients died,
Table II. ROC curve diagnosis.

\begin{tabular}{lcc}
\hline Item & Notch1 & HES1 \\
\hline AUC & 0.732 & 0.766 \\
Std.Error & 0.049 & 0.043 \\
95\% CI & $0.636-0.827$ & $0.682-0.850$ \\
P-value & 0.001 & 0.001 \\
Cut-off & 13.230 & 12.810 \\
Sensitivity (\%) & 93.55 & 82.26 \\
Specificity (\%) & 58.06 & 61.29
\end{tabular}

with a 5-year survival rate of $67.74 \%$; there were 16 patients that died in the high expression group, with a 5-year survival rate of $48.39 \%$. The survival rate of the patients in the low expression group was significantly higher than that of the patients in the high expression group $(\mathrm{P}=0.045)$, as shown in Fig. 4 .

Univariate logistic regression analysis. The patients were divided into the survival group (36 cases) and the mortality group (26 cases) according to their survival conditions. Univariate analysis based on the clinical data of the survival group and mortality group illustrated that there were no significant differences in age, sex and TNM staging between 
A

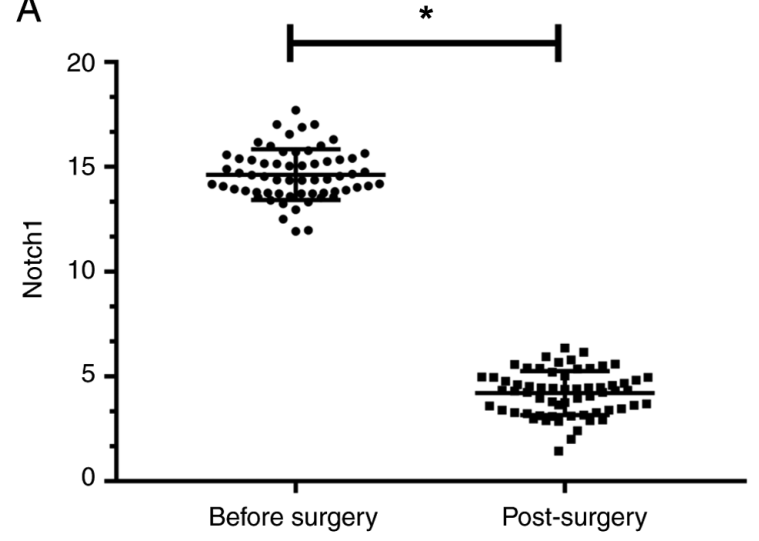

B

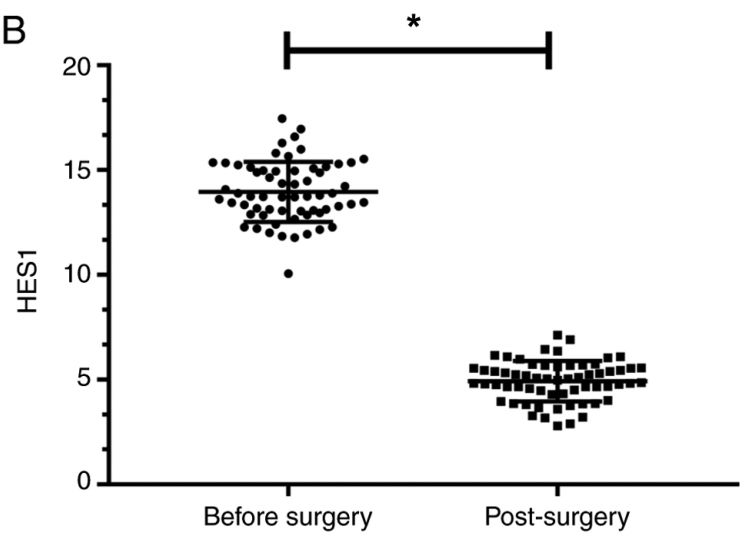

Figure 1. Expression of Notch1 and HES1 in osteosarcoma patients before and after surgery. (A) Expression of Notch1 before and after surgery. (B) Expression of HES1 before and after surgery. The expression levels of Notch1 and HES1 in patients before surgery were significantly higher than those after surgery. ${ }^{*} \mathrm{P}<0.05$.
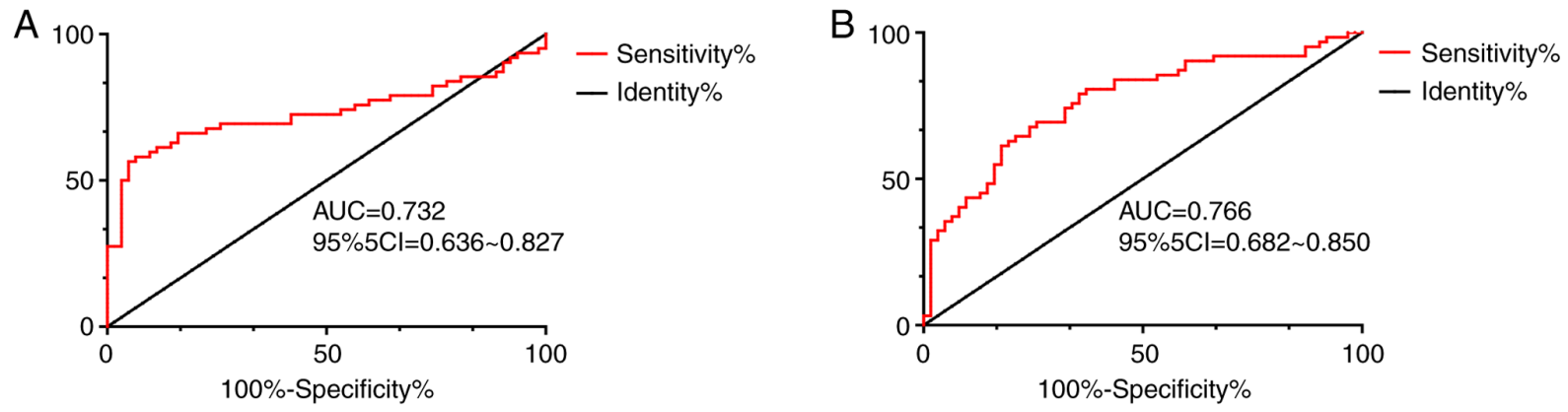

Figure 2. Diagnostic value of Notch1 and HES1 for osteosarcoma. (A) When the cut-off value was 13.230, the sensitivity, specificity and AUC of Notch1 in the diagnosis of osteosarcoma were $93.55 \%, 58.06 \%$ and 0.732 , respectively. (B) When the cut-off value as 12.810 , the sensitivity, specificity and AUC of HES1 in the diagnosis of osteosarcoma were $82.26 \%, 61.29 \%$ and 0.766 , respectively.

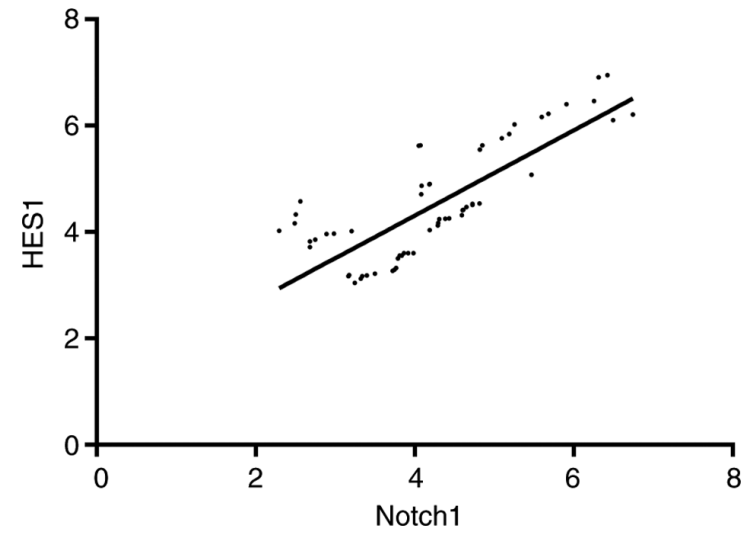

Figure 3. Pearson's correlation analysis. Notch1 expression level positively correlated with the HES1 expression level in osteosarcoma patients $(r=0.795$, $\mathrm{P}<0.001)$.

the groups $(\mathrm{P}>0.05)$. Significant differences were observed for in tumor location, chemotherapy response, tumor size, and Notch1 and HES1 expression $(\mathrm{P}<0.05)$, as shown in Table III.

Multivariate logistic regression analysis. Multivariate difference indicators (tumor location, chemotherapy response and tumor size) were assigned, as shown in Table IV. Subsequently,

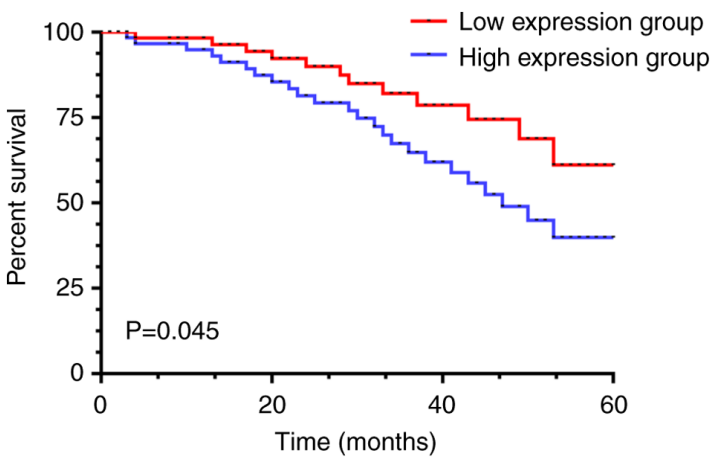

Figure 4. Patients were divided into the high and low expression groups according to the expression levels of Notch1 and HES1, and the 5-year survival rate of the patients was observed. According to the median value of the detection results of the expression levels of Notch1 and HES1, they were divided into Notch1 high expression group $(\geq 16.57)$, HES1 high expression group $(\geq 15.18)$ (31 cases), low expression group $(<16.57)$ and low expression group (<15.18) (31 cases). The 5-year survival rate of patients in the Notch1 and HES1 low expression group was $67.74 \%$, while that of patients in the high expression group was $48.39 \%$. The survival rate of patients in the low expression group was significantly higher than that of patients in the high expression group $(\mathrm{P}=0.045)$.

multivariate logistic regression analysis was performed to confirm tumor location (OR, 3.521; 95\% CI, 1.061-3.183), chemotherapy response (OR, 5.020; 95\% CI, 0.218-0.675), 
Table III. Univariate analysis.

\begin{tabular}{|c|c|c|c|c|}
\hline Clinicopathological features & Survival group $(n=36)$ & Mortality group $(n=26)$ & $\chi^{2}$ or t-test value & P-value \\
\hline Age (years) & & & 0.261 & 0.610 \\
\hline$<20$ & $13(41.94)$ & $15(48.39)$ & & \\
\hline$\geq 20$ & $18(58.06)$ & $16(51.61)$ & & \\
\hline Sex, n $(\%)$ & & & 0.272 & 0.602 \\
\hline Male & $20(64.52)$ & $18(58.06)$ & & \\
\hline Female & $11(35.48)$ & $13(41.94)$ & & \\
\hline Tumor location, n (\%) & & & 5.248 & 0.022 \\
\hline Limbs & $10(32.26)$ & $19(61.29)$ & & \\
\hline Not limbs & $21(67.74)$ & $12(38.71)$ & & \\
\hline Chemotherapy response, $\mathrm{n}(\%)$ & & & 4.239 & 0.040 \\
\hline Adverse reaction & $14(45.15)$ & $22(70.97)$ & & \\
\hline Good reaction & $17(54.84)$ & $9(29.03)$ & & \\
\hline TNM staging, n (\%) & & & 0.369 & 0.544 \\
\hline Stages I-II & $23(74.19)$ & $25(80.65)$ & & \\
\hline Stages III-IV & $8(25.81)$ & $6(19.35)$ & & \\
\hline Tumor size, n (\%) & & & 4.133 & 0.042 \\
\hline$\geq 3 \mathrm{~cm}$ & $12(38.71)$ & $20(64.52)$ & & \\
\hline$<3 \mathrm{~cm}$ & $19(61.29)$ & $11(35.48)$ & & \\
\hline Notch1 expression & $19.03 \pm 2.35$ & $16.07 \pm 1.55$ & 5.597 & 0.001 \\
\hline HES 1 expression & $16.86 \pm 1.53$ & $14.02 \pm 1.32$ & 7.630 & 0.001 \\
\hline
\end{tabular}

Table IV. Assignment table.

\begin{tabular}{ll}
\hline Factor & \\
\hline Tumor location & Limbs, $1 ;$ not limbs, 0 \\
Chemotherapy response & Good, $1 ;$ poor, 0 \\
Tumor size & $\geq 3 \mathrm{~cm}, 1 ;<3 \mathrm{~cm}, 0$ \\
Notch1 expression & Data were continuous variables and were analyzed as original data. \\
HES1 expression & Data were continuous variables and were analyzed as original data. \\
\hline
\end{tabular}

Table V. Multivariate logistic regression analysis.

$95 \% \mathrm{CI}$ of $\operatorname{Exp}(\mathrm{B})$

\begin{tabular}{lccccccc} 
Factor & B & SE & Wals & Sig. & Exp (B) & Lower limit & Upper limit \\
\hline Tumor location & 0.608 & 0.280 & 4.709 & 0.030 & 3.521 & 1.061 & 3.183 \\
Chemotherapy response & -0.958 & 0.288 & 11.056 & 0.001 & 5.020 & 0.218 & 0.675 \\
Tumor size & 0.568 & 0.742 & 4.996 & 0.026 & 3.227 & 1.072 & 2.901 \\
Notch1 expression & 0.721 & 0.239 & 5.705 & 0.002 & 4.019 & 1.467 & 1.353 \\
HES1 expression & 0.856 & 0.423 & 5.512 & 0.031 & 4.629 & 5.727 \\
\hline
\end{tabular}

SE, standard error; Wals, Chi-squared test value; Sig., significance; Exp (B), odds ratio.

tumor size (OR, 3.227; 95\% CI, 1.072-2.901), Notch1 expression (OR, 4.019; 95\% CI, 1.467-4.218) and HES1 expression (OR, 4.629; 95\% CI, 1.353-5.727). Tumor location, chemotherapy response and tumor size, and Notch1 and HES1 expression were independent risk factors for the prognosis of patients, as shown in Table V. 


\section{Discussion}

Osteosarcoma is one of the most common primary malignant bone diseases, which severely threatens the health of children and adolescents (16). It has a high tendency of local invasion and early systemic metastasis, such as lung metastasis $(17,18)$. Its morbidity rate is high, mainly among children and adolescents aged between 10 and 25 years, whose skeleton is growing rapidly, accounting for $70 \%$ of all osteosarcoma cases (19). Osteosarcoma has a high malignancy and a poor prognosis. According to statistics, approximately $85 \%$ of osteosarcoma patients exhibit metastasis (20). Chen et al (21) and Shin et al (22) demonstrated that the 5-year survival rate of non-metastatic patients increased to $55-70 \%$ with the application of high-dose combination chemotherapy. However, the 5-year survival rate of metastatic patients was only 5-20\%. Although the survival rate of osteosarcoma patients has improved, there certain serious issues still exist, including severe side-effects and recurrent or metastatic disease (23). Therefore, it is of utmost importance to identify effective indicators for the diagnosis and prognosis of patients with osteosarcoma.

Notch1 is an evolutionarily conserved ligand-receptor signaling system that regulates cell proliferation, survival, apoptosis and differentiation $(24,25)$. The dysfunction of the Notch1 signaling pathway may lead to abnormal differentiation or undifferentiation, and may eventually lead to the malignant transformation of these cells. Of note, it has been revealed that changes in Notch1 signaling are associated with a number of human cancers (26-28); however, the role of Notch1 in osteosarcoma has yet not been elucidated. HES1 is a highly conserved basic helix-loop-helix transcription inhibitor, which mediates its biological effects by binding to $\mathrm{N}$-cassettes (CACNAG) in the entire genome and recruiting chromatin modification factors to these sites $(29,30)$. HES1 is necessary for organogenesis and development of several species as a component of Notch1 $(31,32)$. However, the molecular function of HES1 in adult tissues remains unclear. Therefore, by investigating the clinical diagnostic values of Notch1 and HES1 in osteosarcoma patients and their influence on prognosis, this may provide the basis for the future clinical diagnosis and treatment of osteosarcoma.

In the present study the expression levels of Notch1 and HES1 in osteosarcoma patients before and after surgery, we first observed. It was found that Notch1 and HES1 in osteosarcoma patients after surgery exhibited a low expression, which differed significantly from that before surgery. This indicated that Notch1 and HES1 may become potential diagnostic and therapeutic targets for osteosarcoma. Therefore, a ROC curve was then drawn and it was found that the areas under the Notch1 and HES1 curves were 0.732 and 0.766, respectively, which were not associated with a high specificity, but with a high sensitivity and were clinical diagnostic indicators of osteosarcoma. Zhang et al (33) found a new regulatory pathway of invasion and metastasis in osteosarcoma, as well as a novel function of the Notch pathway: The regulation of metastasis. As the Notch pathway can be pharmacologically inhibited, these findings suggest possible novel therapeutic strategies with which to reduce the invasion and metastasis of osteosarcoma. Subsequently, Pearson's correlation analysis demonstrated that the expression level of Notch1 positively correlated with the expression level of HES1 in osteosarcoma patients $(r=0.795, \mathrm{P}<0.001)$. The patients were further divided into the high and low expression groups according to the median value of the expression levels of Notch1 and HES1 in osteosarcoma. Observing the 5-year survival rate of the patients, it was found that the 5-year survival rate of the patients in the Notch1 and HES1 high expression groups was $48.39 \%$, and that of the patients in the Notch1 and HES1 low expression groups was $67.74 \%$. The higher the expression levels of Notch1 and HES1, the lower the survival rate of the osteosarcoma patients, suggesting that Notch1 and HES1 may be used as prognostic survival indicators of osteosarcoma patients. Finally, it was found that tumor location, chemotherapy response, tumor size, and Notch1 and HES1 expression were independent prognostic factors of patients through logistic multivariate analysis, which indicated that tumor location, chemotherapy response, tumor size, Notch1, HES1 and may be used as prognostic indicators for patients with osteosarcoma.

The present study preliminarily proved the clinical value of Notch1 and HES1 through the above-mentioned findings. However, there are still certain limitations to this research. First, tissue samples were not collected and basic cell experiments were not performed. Second, no animal experiments were conducted. Thus, the authors aim to conduct further in-depth experimental analyses as soon to confirm and further broaden the findings of the present study.

In conclusion, the present study demonstrated that Notch1 and HES1 were highly expressed in osteosarcoma patients. Notch1 and HES1 as indicators exhibited a good diagnostic efficacy, as shown by ROC curve analysis, and Notch1 and HES1 expression were strongly associated with the occurrence and development of osteosarcoma. Thus, they may prove to be efficient markers for the diagnosis and prognosis of patients with osteosarcoma. These findings may provide future reference and insight into future studies on osteosarcoma.

\section{Acknowledgements}

Not applicable.

\section{Funding}

No funding was received.

\section{Availability of data and materials}

The datasets used and/or analyzed during the present study are available from the corresponding author on reasonable request.

\section{Authors' contributions}

LF, BL, DY and TZ were involved in the conception and design of the study. LF, BL, DY, BW and TZ were responsible for data collection and analysis. $\mathrm{LF}, \mathrm{BW}$ and TZ were responsible for the interpretation of the data and for drafting the manuscript. LF and TZ made revisions from a critical perspective for important intellectual content. All authors have read and confirmed the final manuscript. 


\section{Ethics approval and consent to participate}

The present study was approved by the Ethics Committee of Shandong Cancer Hospital. Signed written informed consents were obtained from the patients and/or parents or guardians.

\section{Patient consent for publication}

Not applicable.

\section{Competing interests}

The authors declare that they have no competing interests.

\section{References}

1. Moore DD and Luu HH: Osteosarcoma. Cancer Treat Res 162 : 65-92, 2014.

2. Harrison DJ, Geller DS, Gill JD, Lewis VO and Gorlick R: Current and future therapeutic approaches for osteosarcoma Expert Rev Anticancer Ther 18: 39-50, 2018.

3. Ottaviani G and Jaffe N: The epidemiology of osteosarcoma Pediatric and adolescent osteosarcoma. Cancer Treat Res 152 3-13, 2009.

4. Lamora A, Talbot J, Bougras G, Amiaud J, Leduc M, Chesneau J, Taurelle J, Stresing V, Le Deley MC, Heymann MF, et al: Overexpression of smad7 blocks primary tumor growth and lung metastasis development in osteosarcoma. Clin Cancer Res 20 5097-5112, 2014.

5. Artavanis-Tsakonas S, Rand MD and Lake RJ: Notch signaling: Cell fate control and signal integration in development. Science 284: 770-776, 1999.

6. Pece S, Serresi M, Santolini E, Capra M, Hulleman E, Galimberti V,Zurrida S, Maisonneuve P, Viale G and Di Fiore PP: Loss of negative regulation by Numb over Notch is relevant to human breast carcinogenesis. J Cell Biol 167: 215-221, 2004.

7. Li Y, Zhang J, Ma D, Zhang L, Si M, Yin H and Li J: Curcumin inhibits proliferation and invasion of osteosarcoma cells through inactivation of Notch-1 signaling. FEBS J 279: 2247-2259, 2012.

8. Diévart A, Beaulieu N and Jolicoeur P: Involvement of Notch1 in the development of mouse mammary tumors. Oncogene 18: 5973-5981, 1999.

9. Garber K: Notch emerges as new cancer drug target. J Natl Cancer Inst 99: 1284-1285, 2007.

10. Wang SC, Lin XL, Wang HY, Qin YJ, Chen L, Li J, Jia JS, Shen HF, Yang S, Xie RY, et al: Hes1 triggers epithelial-mesenchymal transition (EMT)-like cellular marker alterations and promotes invasion and metastasis of nasopharyngeal carcinoma by activating the PTEN/AKT pathway. Oncotarget 6 : 36713-36730, 2015.

11. Candy PA, Phillips MR, Redfern AD, Colley SM, Davidson JA Stuart LM, Wood BA, Zeps N and Leedman PJ: Notch-Induced transcription factors are predictive of survival and 5-fluorouracil response in colorectal cancer patients. Br J Cancer 109 1023-1030, 2013

12. Farnie G, Clarke RB, Spence K, Pinnock N, Brennan K, Anderson NG and Bundred NJ: Novel cell culture technique for primary ductal carcinoma in situ: Role of Notch and epidermal growth factor receptor signaling pathways. J Natl Cancer Inst 99 616-627, 2007.

13. Konishi J, Kawaguchi KS, Vo H, Haruki N, Gonzalez A, Carbone DP and Dang TP: Gamma-secretase inhibitor prevents Notch3 activation and reduces proliferation in human lung cancers. Cancer Res 67: 8051-8057, 2007.
14. Bielack S, Carrle D and Casali PG; ESMO Guidelines Working Group: Osteosarcoma: ESMO clinical recommendations for diagnosis, treatment and follow-up. Ann Oncol 20 (Suppl 4): S137-S139, 2009

15. Ando K, Heymann MF, Stresing V, Mori K, Rédini F and Heymann D: Current therapeutic strategies and novel approaches in osteosarcoma. Cancers (Basel) 5: 591-616, 2013.

16. Sampson VB, Yoo S, Kumar A, Vetter NS and Kolb EA: MicroRNAs and potential targets in osteosarcoma: Review. Front Pediatr 3: 69, 2015.

17. Zhao H, Yao Y, Wang Z, Lin F, Sun Y and Chen P: Therapeutic effect of pirarubicin-based chemotherapy for osteosarcoma patients with lung metastasis. J Chemother 22: 119-124, 2010.

18. He A, Yang X, Huang Y, Feng T, Wang Y, Sun Y, Shen Z and Yao Y: CD133(+) CD44(+) cells mediate in the lung metastasis of osteosarcoma. J Cell Biochem 116: 1719-1729, 2015.

19. Daw NC, Chou AJ, Jaffe N, Rao BN, Billups CA, RodriguezGalindo C, Meyers PA and Huh WW: Recurrent osteosarcoma with a single pulmonary metastasis: A multi-institutional review. Br J Cancer 112: 278-282, 2015.

20. Faisham WI, Mat Saad AZ, Alsaigh LN, Nor Azman MZ, Kamarul Imran M, Biswal BM, Bhavaraju VM, Salzihan MS, Hasnan J, Ezane AM, et al: Prognostic factors and survival rate of osteosarcoma: A single-institution study. Asia Pac J Clin Oncol 13: e104-e110, 2017.

21. Chen J, Ma L and Wei G: Comment on Fu et al:: A systematic review of p53 as a biomarker of survival in patients with osteosarcoma. Tumour Biol 35: 5049-5050, 2014.

22. Shin SH, Jeong HJ, Han I, Cho HS and Kim HS: Osteosarcoma and chondrosarcoma of the shoulder: Site-Specific comparative analysis. Orthopedics 36: e179-e185, 2013.

23. He H, Ni J and Huang J: Molecular mechanisms of chemoresistance in osteosarcoma (Review). Oncol Lett 7: 1352-1362, 2014.

24. Miele L: Notch signaling. Clin Cancer Res 12: 1074-1079, 2006.

25. Chiba S: Notch signaling in stem cell systems. Stem Cells 24 : 2437-2447, 2006

26. Villanueva A, Alsinet C, Yanger K, Hoshida Y, Zong Y, Toffanin S, Rodriguez-Carunchio L, Solé M, Thung S, Stanger BZ and Llovet JM: Notch signaling is activated in human hepatocellular carcinoma and induces tumor formation in mice. Gastroenterology 143: 1660-1669.e7, 2012.

27. Bocchicchio S, Tesone M and Irusta G: Convergence of wnt and notch signaling controls ovarian cancer cell survival. J Cell Physiol 234: 22130-22143, 2019.

28. Lai XX, Li G, Lin B and Yang H: Interference of Notch 1 inhibits the proliferation and invasion of breast cancer cells: Involvement of the $\beta$-catenin signaling pathway. Mol Med Rep 17: 2472-2478, 2018.

29. Takebayashi K, Sasai Y, Sakai Y, Watanabe T, Nakanishi S and Kageyama R: Structure, chromosomal locus, and promoter analysis of the gene encoding the mouse helix-loop-helix factor HES-1. Negative autoregulation through the multiple $\mathrm{N}$ box elements. J Biol Chem 269: 5150-5156, 1994.

30. Sasai Y, Kageyama R, Tagawa Y, Shigemoto R and Nakanishi S: Two mammalian helix-loop-helix factors structurally related to Drosophila hairy and enhancer of split. Genes Dev 6: 2620-2634, 1992.

31. Ishibashi M, Ang SL, Shiota K, Nakanishi S, Kageyama R and Guillemot F: Targeted disruption of mammalian hairy and Enhancer of split homolog-1 (HES-1) leads to up-regulation of neural helix-loop-helix factors, premature neurogenesis, and severe neural tube defects. Genes Dev 9: 3136-3148, 1995.

32. Ohtsuka T, Ishibashi M, Gradwohl G, Nakanishi S, Guillemot F and Kageyama R: Hes1 and Hes5 as notch effectors in mammalian neuronal differentiation. EMBO J 18: 2196-2207, 1999.

33. Zhang P, Yang Y, Zweidler-McKay PA and Hughes DP: Critical role of notch signaling in osteosarcoma invasion and metastasis. Clin Cancer Res 14: 2962-2969, 2008. 